\title{
OFICINAS TERAPÊUTICAS PARA HÁBITOS DE VIDA SAUDÁVEL NO CENTRO DE CONVIVÊNCIAARTHUR BISPO DO ROSÁRIO: RELATO DE EXPERIÊNCIA
}

\author{
Eduardo Caires Damasceno ${ }^{1}$, Amanda Márcia dos Santos Reinaldo²
}

\begin{abstract}
RESUMO: Este trabalho tem como objetivo relatar uma experiência, como acadêmicos de enfermagem nas atividades desenvolvidas em um Centro de Convivência da cidade de Belo Horizonte-MG, vinculadas ao Projeto de Extensão intitulado "Oficinas Terapêuticas para hábitos de vida saudável no Centro de Convivência Arthur Bispo do Rosário". Trata-se de um relato de experiência no qual avaliamos a importância deste tipo de atividade, no âmbito da graduação. Como resultado de nossa observação, análise e discussão conjunta, pudemos constatar os benefícios advindos da participação dos usuários nas atividades que são realizadas neste espaço, tais como: a ampliação das relações sociais e o desenvolvimento de potencialidades no campo da arte. Acreditamos que a manutenção e ampliação destes espaços sejam de suma importância para a eficaz atenção em saúde mental.
\end{abstract}

PALAVRAS-CHAVE: Saúde mental; Reforma psiquiátrica; Reabilitação psicossocial.

\section{THERAPEUTIC WORKSHOPS FOR HEALTHY LIFE HABITS AT THE ARTHUR BISPO DO ROSÁRIO CONVENTION CENTER: EXPERIENCE REPORT}

\begin{abstract}
This study aims to report our experience, as a students, in activities developed at a community Center in Belo Horizonte - MG, Brazil, related to the Extension Project called "Therapeutic Workshops for healthy life habits at the Arthur Bispo do Rosário Convention Center”. In this experience report, we assess the importance of this kind of activity in the context of an undergraduate program. As a result of our observation, analysis and joint discussions, we could observe the benefits that arose from the users' participation in the activities accomplished in this space, such as: the expansion of social relations and the development of potentialities in the field of art. We believe that the maintenance and expansion of these spaces are extremely important for effective mental health care.
\end{abstract}

KEYWORDS: Mental health; Psychiatric reform; Psychosocial rehabilitation.

\section{TALLERES TERAPÉUTICOS PARA HÁBITOS DE VIDA SALUDABLE EN EL CENTRO DE CONVIVENCIAARTHUR BISPO DEL ROSARIO: RELATO DE EXPERIENCIA}

RESUMEN: La finalidad de este trabajo es relatar nuestra experiencia como académicos de enfermería en las actividades desarrolladas en un Centro de Convivencia de la ciudad de Belo Horizonte - MG/Brasil, vinculadas al Proyecto de Extensión intitulado "Talleres Terapéuticos para hábitos de vida saludable en el Centro de Convivencia Arthur Bispo del Rosario". Se trata de un relato de experiencia donde evaluamos la importancia de este tipo de actividad, en el ámbito del curso de graduación. Como resultado de nuestra observación, análisis y discusión conjunta, pudimos constatar los beneficios derivados de la participación de los usuarios en las actividades que son realizadas en este espacio, tales como: la ampliación de las relaciones sociales y el desarrollo de potencialidades en el campo del arte. Creemos que la manutención y ampliación de estos espacios sean de suma importancia para la eficaz atención en salud mental.

PALABRAS CLAVE: Salud mental; Reforma psiquiátrica; Rehabilitación psicosocial.

${ }^{1}$ Acadêmico do Curso de Graduação em Enfermagem. Escola de Enfermagem da Universidade Federal de Minas Gerais-EEUFMG. Bolsista CNPq.

${ }^{2}$ Enfermeira. Doutora em Enfermagem Psiquiátrica. Docente do Departamento de Enfermagem Aplicada da EEUFMG.

Autor correspondente:

Amanda Márcia dos Santos Reinaldo

Av. Prof. Alfredo Balena, 190 - 30130-100 - Belo Horizonte-MG

Recebido: 08/08/08

E-mail: amsreinaldo@enf.ufmg.br.

Aprovado: 20/02/09

Cogitare Enferm 2009 Jan/Mar; 14(1):178-82 


\section{INTRODUÇÃO}

Nas últimas décadas as discussões acerca de cidadania têm sido ampliadas e alcançado parcelas da população que, ao longo da história, se notabilizaram por ficarem à margem da dinâmica da vida social e em consequência, alienadas acerca de seus direitos. Dentre estas, destacamos de modo especial, a de portadores de transtornos mentais.

Nesse panorama recente em nossa sociedade, vale ressaltar que tal ocorrência só se tornou uma realidade com o advento da reforma psiquiátrica. Com ela foram cada vez mais sendo questionadas as abordagens de tratamento que obtinham como resultado a cronificação dos doentes nos manicômios. Esse movimento, que é contínuo, tem visado restabelecer a cidadania da pessoa com transtornos mentais por meio da desconstrução do modelo asilar.

Vários espaços e experiências para a atenção em saúde mental têm sido efetivados nessa perspectiva, tanto como elementos terapêuticos quanto como promotores de reinserção social, por meio de ações que envolvem o trabalho, a geração de renda e a autonomia do sujeito. Mas é digno de nota que até mesmo estas propostas estão sendo constantemente revistas na prática diária, a fim de não se incorrer em uma nova "institucionalização" que, apesar de mais flexível se comparada ao velho modelo asilar, pode vir a criar novos crônicos ${ }^{(1)}$.

Os Centros de Convivência-CC são um destes espaços. Eles se configuram como instituições públicas que integram a rede substitutiva de atenção à saúde mental e que oferecem às pessoas com transtornos mentais espaços de sociabilidade, produção cultural e intervenção na cidade. Estes Centros visam facilitar a construção de laços sociais e a inclusão das pessoas com transtornos mentais através de espaços de convívio e trabalho das diferenças na comunidade. Os Centros de Convivência atuam, prioritariamente, no campo da cultura e não no da saúde e apresentam valor estratégico para efetivar a inclusão social.

A clientela dos CC é composta, sobretudo, mas não exclusivamente, de indivíduos com transtornos mentais. Suas oficinas e atividades coletivas compõem o eixo do seu trabalho. Em vários municípios do país, estes espaços vêm se consolidando como um dispositivo inovador desempenhando um papel significativo na inclusão social das pessoas com transtornos mentais.

Tem-se debatido a viabilidade da expansão desta estratégia de reabilitação psicossocial para todo o país. No entanto, devido a suas características, os Centros de Convivência costumam ser implementados em municípios que já foram capazes de construir minimamente uma rede efetiva de acolhimento e atendimento eficaz aos indivíduos portadores de transtornos mentais severos e persistentes. Sua criação se justifica apenas nas localidades onde a rede substitutiva de atenção à saúde mental conta com cobertura adequada, especialmente de Centros de Atenção Psicossocial ${ }^{(1-2)}$.

Os CC em Belo Horizonte localizam-se hoje em diversas regiões da cidade, e se configuram como palco das oficinas terapêuticas como parte estratégica do processo de reabilitação dos indivíduos em sofrimento mental. Assim, entende-se que as oficinas representam um papel fundamental nas relações com seu público, uma vez que convidam o portador de sofrimento psíquico, não raro submerso em um mundo próprio, a sair de uma posição passiva e assumir um papel ativo de participação e, consequentemente o insere em uma nova esfera de relações ${ }^{(3)}$.

Este trabalho tem como objetivo relatar nossa experiência, como acadêmicos nas atividades desenvolvidas em um centro de convivência da cidade de Belo Horizonte-MG. Essa experiência é fruto da realização de oficinas de autocuidado vinculadas ao Projeto de Extensão intitulado "Oficinas Terapêuticas para hábitos de vida saudável no Centro de Convivência Arthur Bispo do Rosário”.

\section{OFICINAS TERAPÊUTICAS PARA HÁBITOS DE VIDA SAUDÁVEL NO CENTRO DE CONVIVÊNCIA ARTHUR BISPO DO ROSÁRIO}

O Centro de Convivência Arthur Bispo do Rosário foi inaugurado em 1998, em seu endereço atual, tendo ocupado anteriormente um espaço do Hospital Psiquiátrico Raul Soares, também localizado na cidade de Belo Horizonte. Alguns usuários já frequentavam este espaço de convivência e posteriormente, continuaram a frequentá-lo, o que denota a importância do vínculo com profissionais e oficineiros.

O projeto de extensão intitulado "Oficinas Terapêuticas para hábitos de vida saudável no Centro de Convivência Arthur Bispo do Rosário” é coordenado por duas docentes da Escola de Enfermagem da Universidade Federal de Minas Gerais-EEUFMG, sendo uma enfermeira que é do quadro de docentes do Curso de Graduação em Enfermagem da EEUFMG e outra nutricionista que pertence ao quadro do Curso de 
Nutrição, oferecido também por esta Escola. Atualmente conta com a participação de sete discentes (três acadêmicos de enfermagem e quatro de nutrição) e pela gerente do Centro de Convivência Arthur Bispo do Rosário que é psicóloga da Secretaria Municipal de Saúde de Belo Horizonte.

O projeto é registrado como atividade extensionista no Centro de Extensão da EEUFMG (CENEX) e no Sistema Nacional de Extensão-SIEX. A idéia do projeto surgiu após uma visita que a princípio se destinava a abrir um novo campo de estágio para os alunos da disciplina Enfermagem Psiquiátrica da EEUFMG. O que de fato atualmente acontece regularmente.

Durante esse primeiro contato a docente do curso de enfermagem, coordenadora do projeto, após participar de uma das oficinas oferecidas pelo Centro (oficina de comunicação), observou que os presentes apresentavam muitas dúvidas em relação a hábitos de vida saudável e cuidados com a alimentação. A partir dessa observação apresentou um projeto de oficinas, voltadas para esses temas, à gerente do local que aceitou a idéia.

A partir desse diagnóstico inicial foi realizada uma assembléia com os usuários do CC para levantamento de problemas que pudessem ser trabalhados durante as oficinas. Dúvidas em relação à alimentação, cuidados com o corpo, doenças crônicodegenerativas tais como câncer, hábitos de vida saudável, sexualidade entre outros foram temas solicitados para serem discutidos.

O tema alimentação, cuidados com os alimentos e dieta saudável por ter sido recorrente foi o que motivou a parceria entre o curso de enfermagem e o curso de nutrição para realização do projeto. Após aprovação da realização do projeto pela Secretaria Municipal de Saúde de Belo Horizonte, os alunos selecionados para o projeto passaram a fazer um estágio de seis meses no local, com o objetivo de ambientação às atividades ali desenvolvidas e de formação de vínculo com os usuários do Arhur Bispo, como é conhecido por todos.

O estágio serviu para que os alunos também se familiarizassem com a rotina do serviço e participassem de todas as suas atividades culturais (mostras de arte, exposições, oficinas de música, teatro, pintura, bordado, comunicação, entre outras) desenvolvidas pelo Arthur Bispo. Findo o período de estágio os alunos começaram a organizar as oficinas a serem desenvolvidas. Os temas são definidos pelos próprios usuários e para desenvolvê-los são utilizados dois dias na semana. Cada oficina tem duração em média de 2 a 4 horas dependendo do tema a ser abordado. As ações desenvolvidas nas oficinas são registradas em um diário de campo e avaliadas pelos usuários ao seu término.

As oficinas são realizadas pelos alunos e supervisionadas pelos docentes e a gerente do Arthur Bispo. Em relação ao estudo teórico para construção das oficinas os alunos foram orientados sobre a utilização das oficinas terapêuticas no processo de reabilitação psicossocial, por meio da leitura e discussão em grupo da literatura corrente sobre o tema e que também foi utilizada parcialmente nesse relato de experiência ${ }^{(4)}$

O ponto alto deste tipo de atividade, no âmbito da graduação, se dá pela consolidação do conhecimento e desenvolvimento de habilidades e, ao mesmo tempo, possibilita o reconhecimento da importância dos Centros de Convivência como espaços facilitadores de integração social e resgate de cidadania para os indivíduos portadores de transtornos mentais nesses tempos de Reforma Psiquiátrica.

\section{METODOLOGIA}

Trata-se de um relato de experiência elaborado a partir de uma experiência prática e concreta na fase inicial de nosso projeto de extensão - período de adaptação ocorrido nos meses de maio a julho de 2008 no Centro de Convivência Arthur Bispo do Rosário, localizado na cidade de Belo Horizonte/MG.

\section{DISCUSSÃO}

O ser humano é multifacetado e quando se trata de pessoas com transtornos mentais, a própria situação impõe dificuldades na vivência desses aspectos. Ao longo de muitos anos a psiquiatria focalizou apenas a doença, em busca da remissão de sinais e sintomas, pondo de lado o que se poderia oferecer ao paciente, para que este pudesse ultrapassar sua condição ${ }^{(1)}$.

Vários autores enfatizam a importância do pertencimento a um grupo, da convivência e da comunicação com o outro, e do desenvolvimento do pragmatismo. Assim, a função terapêutica das oficinas é dada pela própria convivência que ela instaura, por meio da relação que se estabelece entre oficineiros e usuários do serviço. A convivência, desse modo, apresenta um lugar central no dispositivo terapêutico. Grande parte dos transtornos mentais é marcada por uma forte tendência ao isolamento bem como pela 
dificuldade de se estabelecer vínculos afetivos e sociais. Porém, ainda mesmo que esse isolamento persista no cotidiano, o momento de convivência nas oficinas assume importante valor ${ }^{(1,3)}$.

Tem-se assistido nos últimos vinte anos a uma construção de inúmeras práticas nas quais atividades expressivas, criativas e produtivas, relacionadas a abordagens psicodinâmicas, estéticas e sociais, são convidadas a fazerem parte de um processo de transformação das instituições psiquiátricas ${ }^{(5,6)}$.

Ainda, acerca destas mudanças no modo de se pensar e entender o doente mental e as próprias instituições com seu aparato, Amarante ${ }^{(7)}$ lembra que o processo que visa desinstitucionalizar o doente passa pela operação de colocar entre parênteses a doença mental, não no sentido de sua negação, mas da alternativa por outro olhar, para além da visão estereotipada e classificatória sobre a loucura e o sofrimento.

Em Belo Horizonte apostou-se, a princípio, no processo de reabilitação por meio de oficinas de arte, as quais, hoje, possuem artistas em seu corpo de profissionais, atuando como formadores de opiniões junto à clientela psiquiátrica no caminho da resocialização. E para tanto, lançam mão das múltiplas linguagens que a arte oferece ${ }^{(3)}$.

No Centro de Convivência Arthur Bispo as atividades desenvolvidas são bem variadas, agrupandose em oficinas que envolvem: a) o esforço característico do trabalho; b) atividades expressivas c) atividades recreativas (jogos, festas, passeios, rádio); d) atividades culturais. Outras atividades fora do espaço do centro de convivência têm sido programadas incrementando e ampliando o processo de reinserção social do portador de transtorno mental.

Em nossa experiência temos presenciado a valorização da subjetividade nas oficinas do Arthur Bispo, nas quais, não se evidencia a exploração da mão de obra dos usuários. É digno de nota o fato de que o sofrimento da experiência psicótica, ao se revelar como uma ruptura na comunicação com o mundo faz do exercício das linguagens - sejam elas visuais, auditivas ou corporais -, organizadas em oficinas ou grupos, elementos propiciadores de um cotidiano mais pleno da vida.

Não raro, vários usuários inicialmente apenas integram a oficina, como se constituíssem uma presença ausente, sem participar do fazer do grupo. Todavia, o respeito pela escolha do paciente tem o efeito de, no decorrer do processo, aumentar sua participação nas atividades oferecidas ${ }^{(1)}$.

Pudemos constatar essa idéia em pacientes recém-admitidos no espaço, os quais, a princípio apresentando um comportamento arredio e desconfiado, aos poucos vão se envolvendo nas atividades e com as pessoas ao seu redor. À medida que aumentam a freqüência de visitas ao espaço vão assumindo uma postura ativa na escolha das atividades e oficinas que mais lhes agradam e também passam a se comunicarem de modo espontâneo, expondo idéias, opiniões e desejos.

Quanto aos resultados benéficos advindos da criação dos Centros de Convivência, vários autores têm se referido a eles de modo consistente, atestando a melhoria da qualidade de vida do indivíduo portador de sofrimento mental que freqüenta estes espaços. Muitos deles têm tematizado em relação à validade dos trabalhos nesse campo, afirmando melhora no quadro clínico dos pacientes ${ }^{(1)}$.

Em nossas observações, análises e discussões conjuntas bem como pelos diálogos com os usuários vez por outra, pudemos ouvir e constatar os benefícios advindos da participação deles nas atividades que neste espaço são realizadas, como por exemplo, a ampliação das relações sociais e de espaços de lazer e também o desenvolvimento de potencialidades no campo da arte.

Destacamos também como resultado dessa experiência de cunho acadêmico no âmbito da graduação algumas reflexões suscitadas por nós, dentre elas: a importância deste tipo de experiência, junto à comunidade, no sentido de oportunizar 0 desenvolvimento de habilidades diversas e consolidação do conhecimento teórico no desenvolvimento da prática; o papel e a importância do profissional da saúde mental nos espaços variados de atenção em saúde e, de modo específico, as diversas e não exploradas possibilidades de atuação profissional neste contexto.

Cabe destacar também a necessidade da compreensão do processo saúde/doença como um tema que deve ser discutido fora dos limites da faculdade, envolvendo a comunidade, para que os futuros profissionais da saúde, nesse caso em particular enfermeiros e nutricionistas possam compreender a dinâmica dos serviços de saúde mental e também ao mesmo tempo reavaliar seus conceitos, preconceitos e idéias equivocadas, que, não raro, corroboram para a perpetuação do estigma ao portador de transtorno mental.

\section{CONSIDERAÇÕES FINAIS}


Os Centros de Convivência, no atual contexto da atenção em saúde mental, configuram-se como espaços que desenvolvem atividades de produção artística e cultural. Eles são uma estratégia válida que tem facilitado, sob diversos aspectos, o resgate da cidadania dos indivíduos portadores de transtorno mental.

As oficinas, articulando com o conceito de rede social, têm contribuído para a reabilitação psicossocial por meio da interação social com a comunidade. Não podemos esquecer que os Centros de Convivência também fazem parte da rede de atenção em saúde mental e assim como outros locais (centros comunitários, programa de saúde da família, centros de saúde, associação de moradores), estão sendo subutilizados em seu potencial de reabilitação das pessoas que sofrem com a doença mental e que circulam pelas cidades como seres invisíveis a esses potenciais espaços terapêuticos ainda pouco explorados.

A aproximação dos alunos da graduação dessas atividades também tem como objetivo, ampliar as possibilidades de atuação dos futuros profissionais nessa realidade que se impõe que é a do processo de reestruturação da assistência psiquiátrica a partir do trabalho em diferentes cenários que ainda têm insuficiente visibilidade na comunidade e na rede assistencial como locais terapêuticos, sendo necessário descobrir que espaços são esses e desenvolver suas potencialidades o mais breve possível.

Com a nossa atenção voltada à reabilitação em saúde mental, pudemos ampliar a nossa percepção acerca do impacto que as vivências no Centro de Convivência promovem na vida do indivíduo portador de transtorno mental no sentido de ensejar a melhoria de sua qualidade de vida, reforçando a idéia de que a manutenção e ampliação destes espaços têm se configurado como algo de fundamental importância para a eficaz atenção em saúde mental.

\section{REFERÊNCIAS}

1. Costa CM; Figueiredo AC, orgnizadores. Oficinas terapêuticas em saúde mental. Rio de Janeiro: Contra Capa Livraria; 2008.

2. Amarante P. Loucos pela vida: a trajetória da reforma psiquiátrica no Brasil. Rio de Janeiro: FIOCRUZ; 1995.

3. Amarante P. Uma aventura no manicômio: a trajetória de Franco Basaglia. Hist Cienc Saúde Manguinhos. 1994 Jul/Out 1(1):61-77.

4. Stefanelli MC. Fukuda IMK, Arantes EC. Enfermagem psiquiátrica e suas dimensões assistenciais. Barueri: Manole; 2008.

5. Desviat M. A Reforma psiquiátrica. Rio de janeiro: FIOCRUZ; 1999.

6. Amarante P. O homem e a serpente outras histórias para a loucura e a psiquiatria. Rio de Janeiro: FIOCRUZ; 1996.

7. Amarante P. Ensaios subjetividade, saúde mental, sociedade. Rio de Janeiro: FIOCRUZ; 2000. 